\title{
Lithium abundance in a turnoff halo star on an extreme orbit ${ }^{\star} \star \star \star$ (Research Note)
}

\author{
M. Spite, F. Spite, E. Caffau, and P. Bonifacio
}

\author{
GEPI, Observatoire de Paris, PSL Research University, CNRS, Univ. Paris Diderot, Sorbonne Paris Cité, \\ Observatoire Place Jules Janssen, 92195 Meudon, France \\ e-mail: monique.spite@obspm.fr
}

Received 2 July 2015 / Accepted 29 August 2015

\begin{abstract}
Context. The lithium abundance in turnoff stars of the old population of our Galaxy is remarkably constant in the metallicity interval $-2.8<[\mathrm{Fe} / \mathrm{H}]<-2.0$, defining a plateau. The Li abundance of these turnoff stars is clearly lower than the abundance predicted by the primordial nucleosynthesis in the frame of the standard Big Bang nucleosynthesis. Different scenarios have been proposed for explaining this discrepancy, along with the very low scatter of the lithium abundance around the plateau.

Aims. The recently identified very high velocity star, WISE J0725-2351 appears to belong to the old Galactic population, and appears to be an extreme halo star on a bound, retrograde Galactic orbit. In this paper, we study the abundance ratios and, in particular the lithium abundance, in this star.

Methods. The available spectra (ESO-Very Large Telescope) are analyzed and the abundances of $\mathrm{Li}, \mathrm{C}, \mathrm{Na}, \mathrm{Mg}, \mathrm{Al}, \mathrm{Si}, \mathrm{Ca}, \mathrm{Sc}, \mathrm{Ti}$, $\mathrm{Cr}, \mathrm{Mn}, \mathrm{Fe}, \mathrm{Co}, \mathrm{Ni}, \mathrm{Sr}$ and $\mathrm{Ba}$ are determined.

Results. The abundance ratios in WISE J0725-2351 are those typical of old turnoff stars. The lithium abundance in this star is in close agreement with the lithium abundance found in the metal-poor turnoff stars located at moderate distance from the Sun. This high velocity star confirms, in an extreme case, that the very small scatter of the lithium plateau persists independent of the dynamic and kinematic properties of the stars.
\end{abstract}

Key words. stars: abundances - stars: Population II - Galaxy: evolution - cosmology: observations

\section{Introduction}

Recently, Scholz et al. (2015) have studied a new high proper motion metal-poor turnoff star, with $\mu=267 \mathrm{mas} / \mathrm{yr}$ selected from a high proper motion survey (Luhman 2014), based on observations with the Wide-field Infrared Survey Explorer (WISE; Wright et al. 2010).

These authors obtained low- and medium-resolution spectra of this star: WISE J072543.88-235119.7 (hereafter WISE J0725-2351). Their findings indicate that the star has a high radial velocity, and thus a resulting Galactic rest frame velocity of about $460 \mathrm{~km} \mathrm{~s}^{-1}$, showing that WISE J0725-2351 belongs to the extreme halo and crosses the Galactic disk on a retrograde bound orbit approaching the Sun at a distance of $400 \mathrm{pc}$.

Scholz et al. (2015) obtained spectra with the mediumresolution spectrograph X-Shooter mounted on the ESO Very Large Telescope and deduced a first estimation of the atmospheric parameters of the star, $T_{\text {eff }}=6250 \mathrm{~K}, \log g=4.0$, $[\mathrm{Fe} / \mathrm{H}]=-2.0$, via a comparison with the observed spectrum to a grid of theoretical spectra based on Kurucz models (Castelli \& Kurucz 2004; Munari et al. 2005) computed with different

* Based on observations obtained at the ESO Paranal Observatory, Chile Programmes 093.D-0127, PI: S. Geier and 189.B-0925, PI: S. Trager.

$\star \star$ Table 2 (line by line abundances of the elements) is only available at the CDS via anonymous ftp

to cdsarc.u-strasbg. fr $(130.79 .128 .5)$ or via

http://cdsarc.u-strasbg.fr/viz-bin/qcat?]/A+A/582/A74 temperatures, gravities, and metallicities. The low metallicity of the star shows that it pertains to the old population of the Galaxy. Scholz et al. (2015) also compared the spectrum of WISE J0725-2351 with those of two classical Population II stars, HD 84937 and HD 140283, and found that the spectra are similar and that these stars should be also comparable in age. The age of HD 140283 is estimated to be close to the age of the universe following VandenBerg et al. (2014).

The aim of the present paper is to check the lithium abundance in this extreme halo turnoff star.

\section{Observations and analysis}

The seven X-Shooter spectra of WISE J0725-2351, obtained during the ESO program ID 093.D-0127(A), were retrieved from the ESO archive, combined, and normalized. The resulting spectrum at $670 \mathrm{~nm}$, has a mean resolution of about $R=11000$ and a $\mathrm{S} / \mathrm{N}$ ratio per pixel of more than 500 . It is thus possible to measure rather weak lines and, in particular, the lithium doublet is clearly visible (see Fig. 1) and can be precisely measured.

For the analysis of the star we used OSMARCS model atmospheres (Gustafsson et al. 2008) and the turbospectrum spectral synthesis code (Alvarez \& Plez 1998; Plez 2012).

In a first step we checked the temperature of the star by fitting the $\mathrm{H}_{\alpha}$ profile, which is a very good temperature indicator for turnoff stars (Cayrel 1988; Fuhrmann et al. 1993; van't Veer-Menneret \& Megessier 1996; Barklem et al. 2002; Bonifacio et al. 2007). The theory of Barklem et al. (2000) 


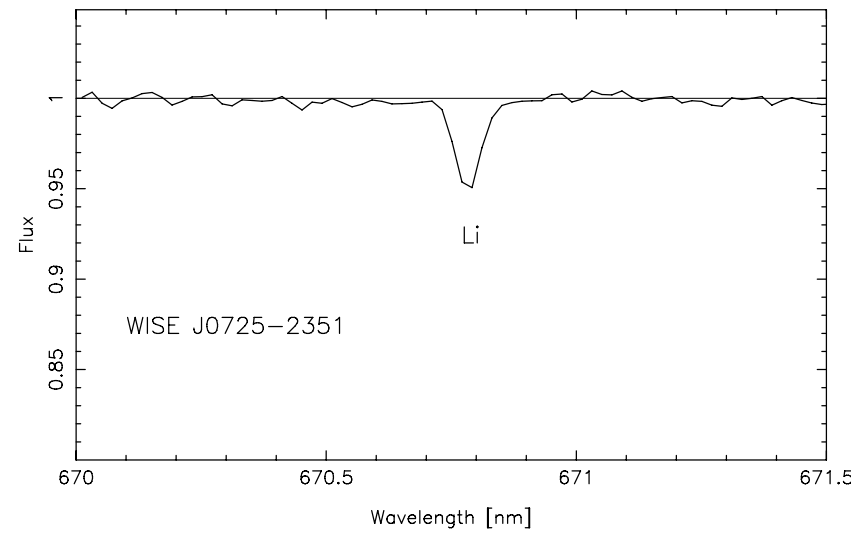

Fig. 1. Observed profile of the lithium feature in WISE J0725-2351.

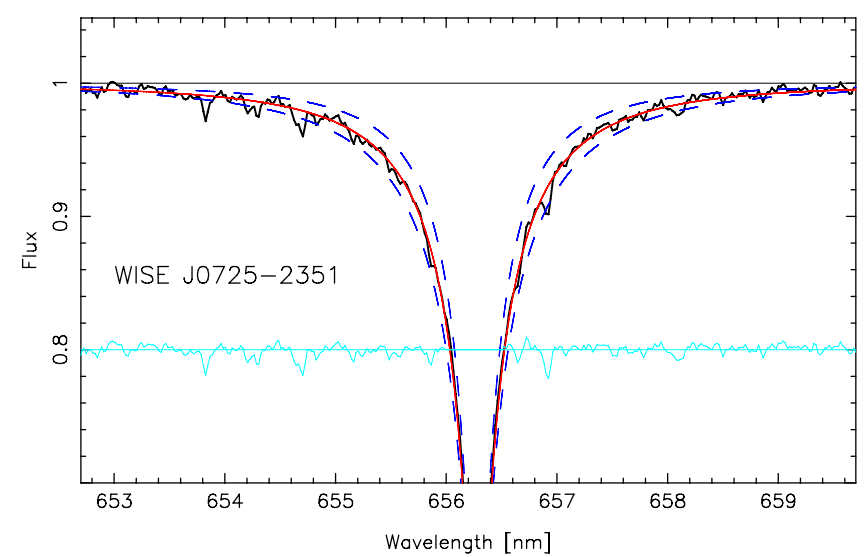

Fig. 2. Observed profile of the $\mathrm{H} \alpha$ line in $\mathrm{CD}-23^{\circ} 5447$ is compared to synthetic spectra computed with $T_{\text {eff }}=5750 \mathrm{~K}$ and $6250 \mathrm{~K}$ (dashed blue lines) and $T_{\text {eff }}=6050 \mathrm{~K}$ (full red line: the best fit).

is used here, via turbospectrum, to treat the hydrogen self-broadening, whereas (Scholz et al. 2015) use the grid of synthetic spectra of Munari et al. (2005) based on the theory of Ali \& Griem (1966) : this theory is known to lead to higher temperatures. We computed theoretical profiles of the $\mathrm{H}_{\alpha}$ line with three values of $T_{\mathrm{eff}}: 5750 \mathrm{~K}, 6000 \mathrm{~K}$, and $6250 \mathrm{~K}$. The best fit is obtained for a temperature of $6050 \mathrm{~K}$; see Fig. 2.

With this temperature, we performed a classical LTE analysis of WISE J0725-2351 using the code MyGIsFOS (Sbordone et al. 2014), as done by Caffau et al. (2013) in the frame of the ESO Large Programme TOPoS. The microturbulence velocity was taken equal to $1.5 \mathrm{~km} \mathrm{~s}^{-1}$, and the surface gravity $\log g=4.2$ was derived from the ionization equilibrium of iron. With these parameters, there is no significant trend in the Fe I abundance with the excitation potential of the line (see Fig. 3). We estimated that that the total error in the adopted temperature is about $100 \mathrm{~K}$, the internal error in the estimation of the gravity is about $0.2 \mathrm{dex}$, and the microturbulent velocity can be constrained within $0.2 \mathrm{~km} \mathrm{~s}^{-1}$.

Following Sitnova et al. (2015), neglecting the NLTE effects in the determination of the gravity from the ionization equilibrium of iron would lead, for metal-poor turnoff stars, to a shift in $\log g$ up to 0.5 dex. The influence of this kind of shift on the abundance of the neutral elements and, in particular, on the lithium abundance $(<0.01 \operatorname{dex}$ for $A(\mathrm{Li}))$ is negligible. The changes in $[\mathrm{X} / \mathrm{Fe}]$ would be also very small if the Fe abundance deduced from the Fe I lines is used as a reference for the neutral species and the abundance deduced from the Fe II lines for

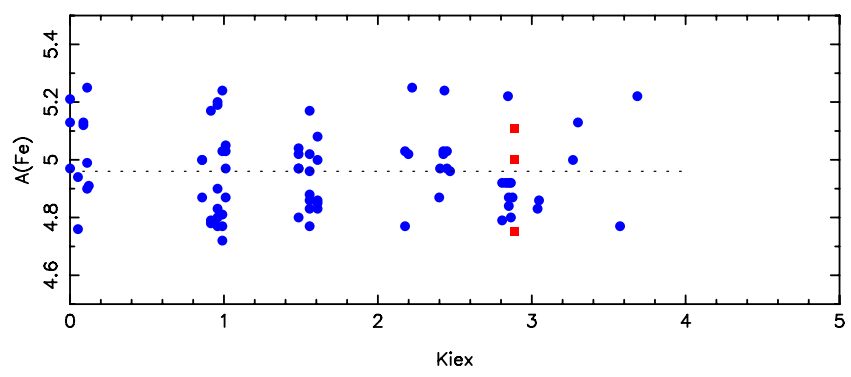

Fig. 3. Comparison of the Fe I (blue dots) and Fe II (red squares) abundances vs. excitation potential for WISE J0725-2351. The adopted model is $T_{\text {eff }}=6050 \mathrm{~K}, \log g=4.2$ and $v_{\mathrm{t}}=1.5 \mathrm{~km} \mathrm{~s}^{-1}$. There is no significant trend of the abundance with the excitation potential of the line.

Table 1. LTE abundances in WISE J0725-2351 obtained with $T_{\text {eff }}=6050 \mathrm{~K}, \log g=4.2$ and $v_{\mathrm{t}}=1.5 \mathrm{~km} \mathrm{~s}^{-1}$.

\begin{tabular}{|c|c|c|c|c|c|c|}
\hline Elem & $\mathrm{N}$ & $A\left(\mathrm{X}_{\odot}\right)$ & $A\left(\mathrm{X}_{\star}\right)$ & {$[\mathrm{X} / \mathrm{H}]$} & {$[\mathrm{X} / \mathrm{Fe}]$} & $\sigma$ \\
\hline $\mathrm{C}(\mathrm{CH})$ & & 8.50 & 6.42 & -2.08 & 0.49 & - \\
\hline $\mathrm{Na} \mathrm{I}$ & 2 & 6.30 & 3.80 & -2.50 & 0.07 & - \\
\hline Mg I & 4 & 7.54 & 5.56 & -1.98 & 0.59 & 0.22 \\
\hline $\mathrm{Al} \mathrm{I}$ & 2 & 6.47 & 3.32 & -3.15 & -0.58 & 0.14 \\
\hline Si I & 1 & 7.52 & 4.98 & -2.54 & -0.03 & - \\
\hline $\mathrm{Ca} I$ & 5 & 6.33 & 4.09 & -2.24 & 0.33 & 0.15 \\
\hline Sc II & 1 & 3.10 & 0.72 & -2.38 & 0.19 & - \\
\hline Ti II & 9 & 4.90 & 2.64 & -2.26 & 0.31 & 0.28 \\
\hline $\mathrm{Cr} \mathrm{I}$ & 4 & 5.64 & 2.78 & -2.86 & -0.29 & 0.23 \\
\hline Mn I & 3 & 5.37 & 2.42 & -2.95 & -0.38 & 0.19 \\
\hline $\mathrm{Fe} \mathrm{I}$ & 77 & 7.52 & 4.95 & -2.57 & 0.00 & 0.20 \\
\hline $\mathrm{Fe}$ II & 3 & 7.52 & 4.95 & -2.57 & 0.00 & 0.26 \\
\hline Co I & 1 & 4.92 & 2.83 & -2.09 & 0.48 & - \\
\hline Ni I & 7 & 6.23 & 3.52 & -2.71 & -0.14 & 0.24 \\
\hline Sr II & 2 & 2.92 & 0.29 & -2.63 & -0.06 & 0.21 \\
\hline Ba II & 1 & 2.17 & -0.20 & -2.37 & 0.20 & - \\
\hline
\end{tabular}

the ionized species (model change would induce similar effects in the abundance of Fe I or Fe II, and XI and X II). To allow an easier comparison to the previous works on similar stars, we prefer not to change the gravity of the model adopted.

The LTE abundances of the different elements are listed in Table 1 (Col. 4), with the number of lines used for the computation (Col. 2) and the adopted solar abundance in Col. 3; $[\mathrm{X} / \mathrm{Fe}]$ is given in Col. 6 and the standard deviation in Col. 7. The largest uncertainty in the abundance determination arises from the uncertainty in the temperature of the star. In general, however, model changes induce similar effects in the abundance of $\mathrm{Fe}$ and the other elements, and as a result they largely cancel out in the ratios [X/Fe] (Cayrel et al. 2004; Bonifacio et al. 2009). As a consequence the uncertainty in $[\mathrm{X} / \mathrm{Fe}]$ is dominated by the random error in the lines. Table 2 (available only at the CDS) lists the main parameters of the lines (wavelength, excitation potential of the lower level, $\log g f$ ) and the resulting abundance of the corresponding element.

The difference between the value of $[\mathrm{Fe} / \mathrm{H}]$ in Table 1 and the value obtained by Scholz et al. (2015) is mainly due to the difference in the adopted temperatures. WISE J0725-2351 is located on the metal-rich edge of the interval of metallicity studied during the ESO Large Programme "First Stars" (Cayrel et al. 2004; Bonifacio et al. 2009). In fact, the abundance ratios are very similar to those found in the star CS 31061-032 (Bonifacio et al. 2009), also on the metal-rich end. 


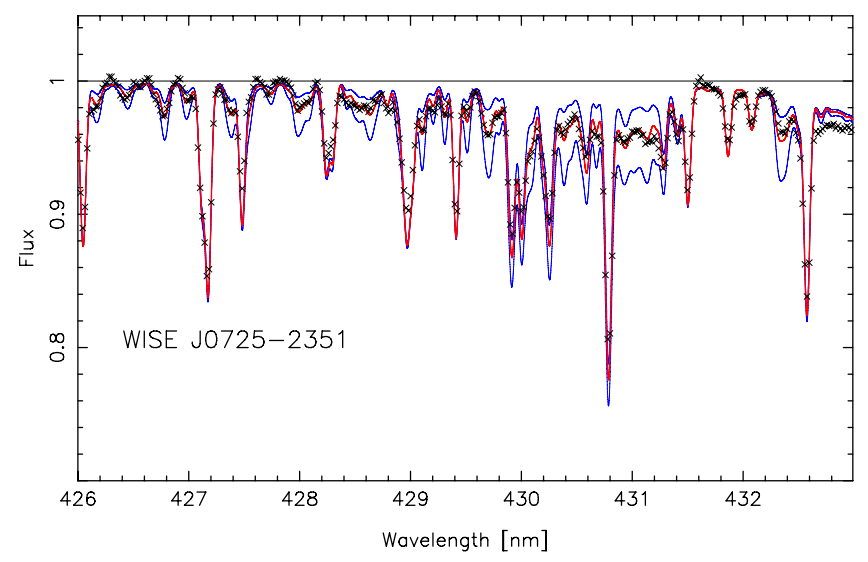

Fig. 4. Observed profile of the $\mathrm{CH}$ band compared to synthetic spectra computed with $A(\mathrm{C})=6.1$ and 6.7 (thin blue lines). The best fit (thick red line) is obtained for $A(\mathrm{C})=6.42$.

As a sanity check of our analysis, we retrieved from the ESO archive an X-Shooter spectrum of HD 84937. It was observed on November 222013 with a 0!'5 slit in the UVB arm and 0.'7 in the VIS arm, yielding resolving power of 7900 in the UVB arm and 11000 in the VIS arm. The exposure time was 6s in both arms, providing $S / N=79$ at $402 \mathrm{~nm}$ and $S / N=71$ at $670 \mathrm{~nm}$. We ran the code MyGIsFOS on these spectra, with fixed effective temperature $6365 \mathrm{~K}, \log g=4.0$, and microturbulent velocity $1.6 \mathrm{~km} \mathrm{~s}^{-1}$ : these atmospheric parameters were derived by Mashonkina et al. (2008) from the analysis of highresolution, high $\mathrm{S} / \mathrm{N}$ spectra. With these parameters they derived $[\mathrm{Fe} / \mathrm{H}]=-2.16$, from the LTE analysis of ten Fe II lines. The MyGIsFOS code, applied to the X-Shooter spectra of our star, provides $[\mathrm{Fe} / \mathrm{H}]_{\mathrm{II}}=-2.23$ with a scatter of $0.13 \mathrm{dex}$ from three $\mathrm{Fe}$ II lines, and $[\mathrm{Fe} / \mathrm{H}]_{\mathrm{I}}=-2.20$ with a scatter of $0.22 \mathrm{dex}$ from 55 Fe I lines. In WISE J0725-2351, the carbon abundance (Fig. 4) is deduced from the profile of the $\mathrm{CH} G$-band. With $[\mathrm{C} / \mathrm{Fe}]=0.49$, WISE J0725-2351 has a carbon abundance very close to the mean value found for turnoff stars in Bonifacio et al. (2009): $\overline{[\mathrm{C} / \mathrm{Fe}]}=0.45 \pm 0.10$.

In Table 1, the abundances of sodium and aluminum are deduced from the resonance lines and are strongly affected by NLTE effects. The NLTE correction for sodium is about -0.34 dex (Andrievsky et al. 2007), and +0.70 dex for aluminum (Andrievsky et al. 2008). As a consequence, in the atmosphere of this star $[\mathrm{Na} / \mathrm{Fe}]_{\mathrm{NLTE}}=-0.27$ and $[\mathrm{Al} / \mathrm{Fe}]_{\mathrm{NLTE}}=+0.12$. These values are in good agreement with the mean values of $[\mathrm{Na} / \mathrm{Fe}]$ and $[\mathrm{Al} / \mathrm{Fe}]$ found in metal-poor stars at this metallicity (Andrievsky et al. 2007, 2008). In particular, $[\mathrm{Al} / \mathrm{Fe}]$ is significantly higher than the low value $\left([\mathrm{Al} / \mathrm{Fe}]_{\text {NLTE }} \sim-0.5\right)$ found by Gehren et al. (2006) in a sample of halo stars, selected from their halo kinematics, at $[\mathrm{Fe} / \mathrm{H}]=-2.5$.

\section{Lithium abundance}

With the atmospheric model obtained in the previous section, we computed the lithium abundance. The equivalent width of the lithium feature is about $30 \mathrm{~m} \AA$, which leads to a lithium abundance $A(\mathrm{Li})=2.17$ (in a logarithmic scale with $A(\mathrm{H})=12$ ). We stress that the lithium abundance does not significantly depend on either the gravity or the microturbulence velocity. Using the fitting formula of Sbordone et al. (2010), which provides the Li abundance taking both NLTE and granulation effects into account (a very small correction), we finally find $A(\mathrm{Li})=2.14$.

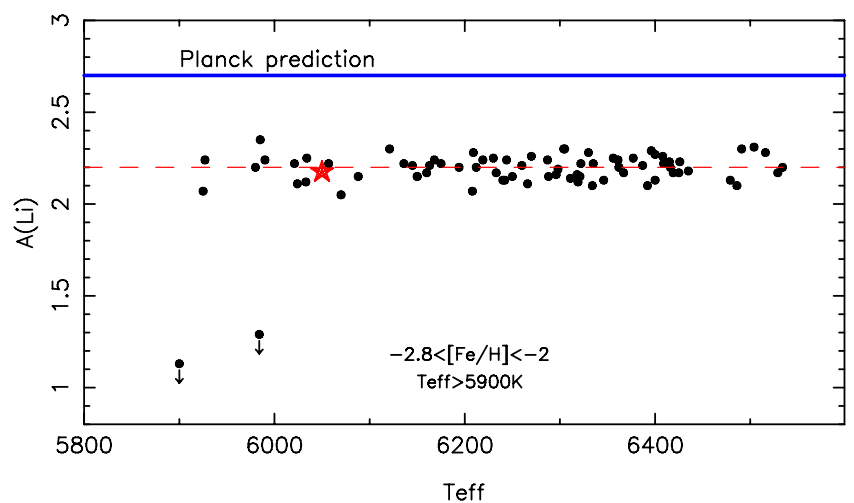

Fig. 5. Lithium abundance vs. $T_{\text {eff }}$ for stars selected for a temperature higher than $5900 \mathrm{~K}$ (without deep convection) and without extreme deficiency $-2.8<[\mathrm{Fe} / \mathrm{H}]<-2.0$. Within these limits, the abundance of lithium is independent of the temperature and the metallicity: $A(\mathrm{Li}) \approx 2.2$ (lithium plateau). The new high proper motion star (red star symbol) is located almost exactly at the mean value of the plateau.

Lithium is a very fragile element destroyed as soon as the temperature exceeds $2.5 \times 10^{6} \mathrm{~K}$. If the convection zone is deep, as in cool stars, lithium is swept along to hot deep layers and is, little by little, destroyed. In Fig. 5, we plotted the lithium abundance as a function of the effective temperature $T_{\text {eff }}$ both for WISE J0725-2351 and for all the field galactic turnoff stars with the Li measurement available in the literature (Charbonnel \& Primas 2005; Asplund et al. 2006; Bonifacio et al. 2007; Aoki et al. 2009; Hosford et al. 2009; Meléndez et al. 2010; Sbordone et al. 2010; Schaeuble \& King 2012). We only selected the stars with temperatures higher than $5900 \mathrm{~K}$, and a metallicity between $[\mathrm{Fe} / \mathrm{H}]=-2$ and $[\mathrm{Fe} / \mathrm{H}]=-2.8$, as in Spite et al. (2012).

The position of the extreme halo star WISE J0725-2351 in Fig. 5 (very close to the mean value) confirms the very small scatter of the lithium abundance in the old galactic stars, in this interval of temperature and metallicity (Spite et al. 2012). The abundance $A(\mathrm{Li})$ does not depend on the stellar kinematic or dynamic properties (see also Boesgaard et al. 2005). At very low metallicity, when $[\mathrm{Fe} / \mathrm{H}]<-2.8$, the lithium abundance in warm metal-poor dwarf stars becomes strongly scattered below the lithium plateau, this sudden melting down of the plateau at very low metallicity (Sbordone et al. 2010) has been discussed in Bonifacio et al. (2015) where different scenarios are proposed. However, WISE J0725-2351 is clearly in the metallicity regime where this effect is not seen.

\section{Conclusion}

The lithium abundance of WISE J0725-2351 has been carefully determined: $A(\mathrm{Li})=2.17$ in LTE and $A(\mathrm{Li})=2.14$ in 3D-NLTE. This recently discovered high velocity star with a retrograde orbit belongs to the extreme halo according to Scholz et al. (2015). This value confirms the low scatter of the lithium abundance (around the mean value $(A(\mathrm{Li})=2.2)$ in the turnoff metal-poor stars with a metallicity higher than $[\mathrm{Fe} / \mathrm{H}]=-2.8$, independent of the kinematic or dynamic properties of the stars (cf. Boesgaard et al. 2005).

A lithium abundance similar to the mean value of the plateau has been also found in the turnoff stars of the galactic globular clusters, M 92 and NGC 6397 (Bonifacio 2002; Bonifacio et al. 2002); in a globular cluster $\omega$ Cen, considered a remnant of a dwarf galaxy (Monaco et al. 2010); and in the globular cluster M 54 (Mucciarelli et al. 2014), pertaining to the Sagittarius 
dSph galaxy. As a consequence, the mean lithium abundance in various old populations is significantly lower than the value predicted by the primordial nucleosynthesis after the Planck measurements (see, e.g., Planck Collaboration XIII 2015; Fields et al. 2014; Cyburt et al. 2015). Different scenarios concerning the formation of the early metal poor stars have been proposed to explain this difference (e.g., Molaro 2012; Fu et al. 2015; Bonifacio et al. 2015).

The chemical composition of WISE J0725-2351 resembles that of the average halo star in spite of its extreme kinematics. This suggests that the star has been formed "in situ". Nevertheless one can not rule out the possibility that it may have been accreted from a Milky Way satellite. In fact, at these low metallicities, some satellites have abundances that are remarkably similar to halo stars, in particular some stars show the typical $\alpha /$ Fe enhancement, for example, in the dwarf galaxy Carina (Koch et al. 2008; Lemasle et al. 2012; Venn et al. 2012), and in several of the ultra-faint dwarfs (François et al., in prep.). The orbit of WISE J0725-2351 could suggest that this star was born outside of the Galaxy and was later accreted. In this case, it could be one more indication of the universality of lithium abundance in the early universe.

Acknowledgements. This work has been supported by the "Programme National de Physique Stellaire" (CNRS-INSU), and it has made use of SIMBAD (CDS, Strasbourg). E.C. is grateful to the FONDATION MERAC for funding her fellowship.

\section{References}

Ali, A. W., \& Griem, H. R. 1966, Phys. Rev., 144, 366

Alvarez, R., \& Plez, B. 1998, A\&A, 330, 1109

Andrievsky, S. M., Spite, M., Korotin, S. A., et al. 2007, A\&A, 464, 1081

Andrievsky, S. M., Spite, M., Korotin, S. A., et al. 2008, A\&A, 481, 481

Aoki, W., Barklem, P. S., Beers, T. C., et al. 2009, ApJ, 698, 1803

Asplund, M., Lambert, D. L., Nissen, P. E., Primas, F., \& Smith, V. V. 2006, ApJ, 644, 229

Barklem, P. S., Piskunov, N., \& O’Mara, B. J. 2000, A\&A, 363, 1091

Barklem, P. S., Stempels, H. C., Allende Prieto, C., et al. 2002, A\&A, 385, 951

Boesgaard, A. M., Stephens, A., \& Deliyannis, C. P. 2005, ApJ, 633, 398
Bonifacio, P. 2002, A\&A, 395, 515

Bonifacio, P., Pasquini, L., Spite, F., et al. 2002, A\&A, 390, 91

Bonifacio, P., Molaro, P., Sivarani, T., et al. 2007, A\&A, 462, 851

Bonifacio, P., Spite, M., Cayrel, R., et al. 2009, A\&A, 501, 519

Bonifacio, P., Caffau, E., \& Spite, M. 2015, A\&A, 579, A28

Caffau, E., Bonifacio, P., Sbordone, L., et al. 2013, A\&A, 560, A71

Castelli, F., \& Kurucz, R. L. 2004, Arxiv e-prints [arXiv:astro-ph/0405087]

Cayrel, R. 1988, The Impact of very high S/N Spectroscopy in Stellar Physics, IAU Symp., 132, 345

Cayrel, R., Depagne, E., Spite, M., et al. 2004, A\&A, 416, 1117

Charbonnel, C., \& Primas, F. 2005, A\&A, 442961

Cyburt, R. H., Fields, B. D., Olive, K. A., \& Yeh, T.-H. 2015, Arxiv e-prints [arXiv: 1505.01076]

Fields, B., Molaro, P., \& Sarkar, S. 2014, Arxiv e-prints [arXiv: 1412.1408]

Fu, X., Bressan, A., Molaro, P., \& Marigo, P. 2015, MNRAS, 452, 3256

Fuhrmann, K., Axer, M., \& Gheren, T. 1993, A\&A, 271, 451

Gehren, T., Shi, J. R., Zhang, H. W., et al. 2006, A\&A, 451, 1065

Gustafsson, B., Edvardsson, B., Eriksson, K., et al. 2008, A\&A, 486, 951

Hosford, A., Ryan, S. G., García Pérez, A. E., Norris, J. E., \& Olive, K. A. 2009, A\&A, 493, 601

Koch, A., Grebel, E. K., Gilmore, G. F., et al. 2008, AJ, 135, 1580

Lemasle, B., Hill, V., Tolstoy, E., et al. 2012, A\&A, 538, A100

Luhman, K. L. 2014, ApJ, 781, 4

Mashonkina, L., Zhao, G., Gehren, T., et al. 2008, A\&A, 478, 529

Meléndez, J., Casagrande, L., Ramírez, I., Asplund, M., \& Schuster, W. J. 2010, A\&A, 515, L3

Molaro, P., Bressan, A., Barbieri, M., Marigo, P., \& Zaggia, S. 2012, MSAIS, 22,233

Monaco, L., Bonifacio, P., Sbordone, L., Villanova, S., \& Pancino, E. 2010, A\&A, 519, A3

Munari, U., Sordo, R., Castelli, F., \& Zwitter, T. 2005, A\&A, 442, 1127

Mucciarelli, A., Salaris, M., Bonifacio, P., Monaco, L., \& Villanova, S. 2014, MNRAS, 444, 1812

Planck Collaboration XIII. 2015, A\&A, submitted [arXiv: 1506.07135]

Plez, B. 2012, record ascl:05004P http://adsabs.harvard.edu/abs/ 2012ascl. soft05004P

Sbordone, L., Bonifacio, P., Caffau , E., et al. 2010, A\&A, 522, A26

Sbordone, L., Caffau, E., Bonifacio, P., \& Duffau, S. 2014, A\&A, 564, A109

Schaeuble, M., \& King, J. R. 2012, PASP, 124, 164

Scholz, R.-D., Heber, U., Heuser, C., et al. 2015, A\&A, 574, A96

Sitnova, T., Zhao, G., Mashonkina, L., et al. 2015, ApJ, 808, 148

Spite, M., Spite, F., \& Bonifacio, P. 2012, Mem. Soc. Astron. It. Supp., 22, 9

Vandenberg, D. A., Bond, H. E., Nelan, E. P., et al. 2014, ApJ, 792, 110

van't Veer-Menneret, C., \& Megessier, C. 1996, A\&A, 309, 879

Venn, K. A., Shetrone, M. D., Irwin, M. J., et al. 2012, ApJ, 751, 102

Wright, E. L., Eisenhardt, P. R., Mainzer, A. K., et al. 2010, AJ, 140, 1868 